\title{
Diabetic Ketoacidosis Treatment Outcome and Associated Factors Among Adult Patients Admitted to Medical Wards of Adama Hospital Medical College, Oromia, Ethiopia
}

\author{
Dagim Assefa Kassaye ${ }^{1}$, Worku Dugassa Girsha ${ }^{2}$, Godana Jarso Guto ${ }^{1}$, Haji Aman Deybasso ${ }^{2}$ \\ ${ }^{1}$ Department of Internal Medicine, Adama Hospital Medical College, Adama, Ethiopia \\ ${ }^{2}$ Department of Public Health, Adama Hospital Medical College, Adama, Ethiopia
}

Email address:

kalkidandag@gmail.com(D. A. Kassaye), dugassaworku@gmail.com(W. D. Girsha), godana.jarso@gmail.com(G. J. Guto), hajia.aman9@gmail.com(H.A. Deybasso)

\section{To cite this article:}

Dagim Assefa Kassaye, Worku Dugassa Girsha, Godana Jarso Guto, Haji Aman Deybasso. Diabetic Ketoacidosis Treatment Outcome and Associated Factors Among Adult Patients Admitted to Medical Wards of Adama Hospital Medical College, Oromia, Ethiopia. American Journal of Internal Medicine. Vol. 6, No. 2, 2018, pp. 34-42. doi: 10.11648/j.ajim.20180602.12

Received: December 1, 2017; Accepted: April 11, 2018; Published: May 19, 2018

\begin{abstract}
Diabetic Ketoacidosis (DKA) is one of the most serious acute complications of diabetes mellitus. The mortality rate remains high in developing countries and among misdiagnosed and treated patients. Therefore targeting early diagnosis and effective treatment programs is vital to save the life of patients. The aim of the study is to assess diabetic ketoacidosis treatment outcome and associated factors among adult patients at Adama Hospital Medical College emergency department and medical wards, Oromia region, Ethiopia. Cross sectional study based on record review of 357 adult diabetic ketoacidosis patients was selected using simple random sampling. Data was collected using checklist from medical registrations. The data was entered and analyzed using SPSS version 20 and EPI-info version 7 statistical packages. Majority of the patients $(65.30 \%)$ had two or more episodes of diabetic ketoacidosis and the main reasons for recurrent diabetic ketoacidosis were infectious illness $(69.50 \%)$ and insulin discontinuation $(14.80 \%)$. Regarding treatment outcomes of diabetic ketoacidosis patients, majority of them $(84.90 \%)$ discharged with improved. Those diabetic ketoacidosis patients treated with more than six liters fluid replacement in the 1st 24 hours had better treatment outcome by two times as compared to less than three liters fluid replacement $(A O R=2.41(1.58-10.02)$. Similarly, those patients who got more than sixty international unit insulin doses in the 1 st $24 \mathrm{hrs}$ had better treatment outcome by ten times $(\mathrm{AOR}=10.68(3.88-20.64))$ when compared to less than forty international unit insulin administration. In addition, DKA patients who got supplemental potassium showed five times treatment outcome improvement $(\mathrm{AOR}=5.30(2.11-13.32)$ than for those potassium replacement was not done. Even if majority of diabetic ketoacidosis patients treated at Adama Hospital Medical College emergency room and medical wards were discharged with improvement, early treatment of infection, ample fluid replacement and insulin dose adjustment during illness need to be encouraged.
\end{abstract}

Keywords: Diabetic Ketoacidosis, Treatment Outcome, Associated Factors, Adama Hospital Medical College

\section{Introduction}

Diabetic ketoacidosis is a potentially life threatening complication of Diabetes Mellitus [1]. It is characterized by hyperglycaemia, dehydration, metabolic acidosis and ketonuria. The criteria for the diagnosis of DKA includes a blood sugar $>14.0 \mathrm{mmol} / \mathrm{l}$, presence of urinary or plasma ketones, a $\mathrm{pH}<7.3$ and a serum bicarbonate of less than 18 $\mathrm{mmol} / 1[2-4]$.
Diabetic ketoacidosis primarily occurs in patients with Type I diabetes mellitus but it is also being recognized in Type II diabetics $[5,6]$. The incidence of DKA is estimated at between 4.6 and 8 episodes per 100 patient years of diabetes. The most occurrence ages of DKA are between the $18-44$ years $(56 \%)$, followed by $45-65$ years $(24 \%)$ and only $18 \%$ of patients $<20$ years of age [2]. 
The most common precipitating event for the development of DKA is infection, which accounts up to $30 \%$ of cases. The second most common precipitating event worldwide is noncompliance with treatment $15 \%$. The third most common cause is the first manifestation of new onset diabetes (5$15 \%)$. Other common precipitating events include other stresses (myocardial infection, alcohol, pancreatitis, drugs) $[1,3]$.

Diabetic ketoacidosis usually develops slowly, but when vomiting occurs, this life-threatening condition can develop in a few hours $[7,8]$. There is a wide spectrum of severity of illness in patients presenting with DKA $[9,10]$. Classically patients present with a history of thirst, polyuria and polydipsia although these are not invariably present. Other symptoms may include: weakness and lethargy, nausea and vomiting, abdominal pain, weight loss. Common general physical signs are evidence of dehydration, tachycardia and hypotension, kussmaul respiration (deep rapid respiration to provide respiratory compensation for metabolic acidosis), ketotic breath (fruity acetone smell due to exhaled ketones), temperature is usually normal or low even in the presence of an underlying infection, altered consciousness and confusion. [11-13].

An understanding of the pathophysiology of the conditions aids appropriate treatment [14]. Initial assessment must involve a rapid assessment of airway, breathing and circulation and obtaining sufficient intravenous access. Initial investigations are aimed at confirming the diagnosis and identifying underlying causes [15]. The basic principles of DKA management include rapid restoration of adequate circulation and perfusion with isotonic intravenous fluids, gradual rehydration and restoration of depleted electrolytes, insulin to reverse ketosis and hyperglycaemia and regular monitoring of clinical signs and laboratory tests to detect and treat complications $[16,17]$.

Diabetes mellitus is the commonest of all metabolic diseases all over the world. The worldwide prevalence of diabetes mellitus has increased dramatically over the past decades from an estimated 30 million cases in 1985 to 177 million in 2000. A recent estimate suggested that diabetes mellitus with complications was the 5th leading cause of death worldwide and is responsible for almost 3 million deaths annually $[5,18,19]$.

In Africa the mortality of DKA is unacceptably high with a reported death rate of 26 to $29 \%$ in studies from Kenya, Tanzania and Ghana. In a Kenyan study, $34 \%$ of DKA events were due to missed insulin injections, $23.4 \%$ to overt infection and only $6.4 \%$ had both infection and missed insulin injections $[4,6]$.

In Ethiopia the prevalence of DKA is increasing for the last two to three decades becoming a major economic problem in drug use and bed occupancy [20, 21]. The control of diabetes and prevention of ketoacidosis in Ethiopian patients is hampered by socioeconomic factors, particularly the cost and unreliability of insulin supplies [22-24].

Diabetes mellitus leads to acute and chronic complications [25]. The acute complications include diabetic ketoacidosis
(DKA), hyperosmolar hyperglycemic state (HHS), and hypoglycemia during treatment and the chronic complications are neuropathy, nephropathy-thy, retinopathy, ischemic heart disease, myocardial infarction, stroke, peripheral arterial disease, impotence and so on [26, 27].

Cerebral oedema remains the most common cause of mortality, particularly in young children and adolescents [9]. In the adult population, hypokalaemia, adult respiratory distress syndrome/ acute lung injury and co-morbid states such as pneumonia, acute myocardial infarction and sepsis are all associated with increased mortality [28, 29].

Diabetic ketoacidosis is a serious conditions that requiring immediate intervention. Diabetic ketoacidosis and the hyperosmolar hyperglycemic state are the two most serious acute metabolic complications of diabetes. DKA is responsible for more than 500,000 hospital days per year at an estimated annual direct medical expense and indirect cost of 2.4 billion USD [30, 31].

Diabetic ketoacidosis is a medical emergency with a significant morbidity and mortality. It should be diagnosed promptly and managed intensively by experienced staff. A fixed-rate, weight based intravenous insulin infusion should be used with bedside measurement of metabolic changes. The specialist diabetes team should be involved as soon as possible and ideally within 24 hours; this has been demonstrated to be associated with a better patient experience and reduced length of stay [32, 33].

In current Ethiopian health care setup, DKA has not been well documented. However, with the current trend of transition from communicable to non-communicable disease, the cases are projecting. Many patients with diabetes keep dying from DKA every year because of misdiagnosis and treatment. Therefore targeting early diagnosis and treatment of effective programs is vital to save the life of patients [20, 21].

Early clinical diagnosis with management like fluid therapy and insulin administration shows significant better outcome and fewer recurrences. DKA can be controlled in a period of 12-36 hours with an appropriate treatment. Thus, complications can be prevented and reduction in mortality rates can be achieved [30].

Even though the cases of DKA are increasing in the health care facilities including the study area, little is known or very little study was done on DKA treatment outcome and their risk factors. Therefore, the study helps health workers and responsible bodies working on the management of DKA to develop uniform treatment guideline or protocol and for planning purpose. The information gained from the study also helps the patients towards prevention methods of DKA. Furthermore the finding of the study was used as a base line data for those who are interested in carrying out further study. Hence, we intended to seek DKA treatment outcome and associated factors among adult DKA patients at Adama Hospital Medical College emergency department and medical wards, Oromia region, Ethiopia. 


\section{Methods and Materials}

\subsection{Study Design and Setting}

Facility based retrospective cross-sectional study design was conducted among DKA patients admitted to Adama Hospital Medical College emergency department and medical wards. The college is found in Adama town located in East Showa Zone about 99 kilometers from the capital city of Ethiopia. The college currently teaches different fields of study like for under graduate medical students, residency programs in internal medicine and others. The total catchments population is about 4,690,000 and the hospital has 200 beds. The college runs several medical outpatient services, including the diabetic follow up clinic which holds on Wednesday and Thursday's weekly program. The weekly diabetic follow-up clinic gives service to 250-300 patients per months. On average around 300 DKA patients are admitted to emergency department and medical wards of the college per year. The study period was a three years retrospective chart review from September 01/2014 to August $30 / 2017$.

\subsection{Sampling Methods}

After listing all complete charts of DKA patients admitted to Adama Hospital Medical College emergency department and medical wards from September 01/2014 to August $30 / 2017$, the required sample was selected using simple random sampling.

\subsection{Sample Size Determination}

To determine the sample size for the study, the following assumptions was considered: According to study done on acute management of diabetic ketoacidosis in Tikur Ambesa Hospital's Adult Emergency department and medical wards, $51.8 \%$ the patients was discharged with improvement (22), Level of confidence, $95 \%,(\mathrm{Z} \alpha / 2)=1.96$, A $5 \%$ margin of error $(\mathrm{d}=0.05)$, then sample size was calculated by using the formula estimate of single population proportion:

$$
\begin{aligned}
& \mathrm{N}=\frac{(2 \alpha / 2)^{2} p(1-p)}{d^{2}} \\
& \mathrm{n}=\frac{1.96^{2} * 0.52 * 0.48}{0.05^{2}}
\end{aligned}
$$

(According to health sector transformation plan of Ethiopia, diabetic Mellitus (DM) accounted around 2\% of the total population and around 5\% DM patients may develop DKA, therefore the estimated DKA patients in the catchment area are around 5000). Accordingly, using finite population correction formula,

$$
\mathrm{n}=\frac{\mathrm{n}_{0}}{\left(1+\frac{\mathrm{n}_{0}}{\mathrm{~N}}\right)}=384 /(1+(384 / 5000))=357
$$

Where, $n=$ final sample size, $n_{0}=$ calculated sample size ${ }_{N}=$ estimated cases in the population, then final corrected sample size obtained for the study was 357 .

\subsection{Exclusion Criteria}

An incomplete chart and age less than 18 years was excluded from the study.

\subsection{Operational Definitions}

Standard protocols for DKA treatment:

1. Fluid replacement: Estimated fluid deficit is 5-7 liters. Correct with normal saline (NS), 2liters in the first 2 hours, then the remainder over the next 22 hours.

2. Insulin administration: Intravenous infusion (IV) bolus of $0.1 \mathrm{IU} / \mathrm{Kg}$ regular insulin followed by an intravenous infusion of $0.1 \mathrm{IU} / \mathrm{kg} / \mathrm{h}$

3. Acid/base $-\mathrm{pH}$ will climb with plasma expansion and insulin administration. Use small amounts of sodium bicarbonate only for severe academia $(\mathrm{pH}<6.9)$.

4. Electrolytes replacement: Close monitoring and correction of serum potassium is critical. (If serum potassium is less than 3.5 , correct hypokalemia before any insulin is given.)

5. Search for cause: Seek to determine underlying precipitant, such as treatment non-adherence, infection, myocardial infarction, etc. $[1,5]$.

Treatment outcome of DKA

1. Showed improvement and discharged out of DKA

2. Not showed improvement (referred for better care or dead in hospital because of DKA complications)

\subsection{Ethical Consideration}

Ethical clearance was obtained from Institutional Review board of the college. Permission letter was written to the medical ward. Orientation was given to medical staff working in the medical wards and main medical record office of Adama hospital medical college. In order to protect the confidentiality, the name of each patient was not written on checklist. The information gained from the study was used only for the purpose of research.

\subsection{Data Collection Techniques}

The data for both independent and outcome variables was collected from medical records using check list adapted from various related studies as well as international and national diabetic association treatment guidelines. Two-day training was given for data collectors regarding data collection technique. Pretesting of checklist on $5 \%$ of the total sample size was undergone in the hospital to identify potential problems that will arise during the actual data collection period and identified problems was amended accordingly. The data collection process was checked daily for accuracy, consistency, and completeness by supervisor. Furthermore, the supervisor and the principal investigator were given feedback and correction regarding the collected data on a daily basis to the data collectors. 


\subsection{Data Processing and Analysis}

Data was checked for completeness, consistency and entered in to epi info version7. Then data was imported in to statistical package for social sciences (SPSS) software Version 21 for data processing and analysis. In the analysis process, frequency distribution of variables was worked out in order to describe them. Independent variables which had association with the outcome variable in the bivariate logistic regression and those with $\mathrm{P}$ _value of $<0.25$ was considered a candidate for the final logistic regression model. Adjusted odds ratio (AOR) of $95 \%$ confidence interval was used to assess the association between independent and dependent variables.

\section{Results}

Of the 357 reviewed charts, more than half $188(57.70 \%)$ were males and $169(47.30 \%)$ were females. The mean ( \pm SD) age of the patients was $39.25 \pm 14.53$ years and the majority (41.73) of them was in the age group 18-30. Nearly half $183(51.30 \%)$ of the patients are poorly controlled type 2 with hyperglycemia and ketosis or ketone prone ones. The majority of patients, 282 (78.99), had been diabetics for less than 5 years. Regarding to their treatment regimen for DM, almost all of them were on subcutaneous insulin 336 (94.10) (Table 1).

Table 1. Socio-demographic characteristics and history of DKA patients admitted to AHMC emergency department and medical wards, Oromia region, Ethiopia, 2017.

\begin{tabular}{ll}
\hline Variables & Frequency (\%) \\
\hline Sex & $188(57.70)$ \\
Male & $169(47.30)$ \\
Female & $39.25 \pm 14.53$ \\
Age mean $\pm(\mathrm{SD})$ years & \\
Age group (years) & $149(41.73)$ \\
$18-30$ & $41(11.48)$ \\
$30.1-40$ & $86(24.08)$ \\
$40.1-50$ & $53(14.45)$ \\
$50.1-60$ & $28(7.84)$ \\
$>60$ & \\
Type of DM & $183(51.30)$ \\
DM-1 & $174(48.70)$ \\
DM-2 & \\
Duration of DM (years) & $282(78.99)$ \\
$1-5$ years & $75(21.01)$ \\
$>5$ years & \\
Treatment regimen for DM & $21(5.90)$ \\
Oral antidiabetic & $336(94.10)$ \\
Insulin & \\
\hline
\end{tabular}

Among the 357 patients in the study, 187 (52.38\%) had one or more of the chronic complications, the major ones being hypertension in $100(29.85 \%)$, diabetic foot ulcer in 61 (18.21\%), sexual dysfunctions in $46(13.73 \%)$. Concerning acute diabetic complication, all of the patients were experienced at least one acute co-morbidities, where urinary tract infection was the commonest accounting for 209 $(22.59 \%)$ followed by respiratory tract infection 192 (20.76\%) (Table 2).
Table 2. Distribution of chronic and acute complications among adult DKA patients admitted to AHMC emergency department and medical wards, Oromia region, Ethiopia, 2017.

\begin{tabular}{ll}
\hline Variable & Frequency (\%) \\
\hline Chronic co-morbidities & $100(29.85)$ \\
Hypertension & $61(18.21)$ \\
Diabetic foot ulcer & $46(13.73)$ \\
Sexual dysfunctions & $32(9.55)$ \\
Diabetic Retinopathy & $28(8.36)$ \\
Diabetic Neuropathy & $27(8.06)$ \\
Periodontal lesions & $21(6.27)$ \\
Cardiovascular Disorder & $14(4.18)$ \\
Renal Disorder & $6(1.79)$ \\
Dyslipidemia & $335(100)$ \\
Total & \\
Acute co-morbidity & $209(22.59)$ \\
Urinary tract infection & $192(20.76)$ \\
Respiratory tract infection & $121(13.08)$ \\
Acute gastroenteritis & $119(12.86)$ \\
Sepsis of any origin & $87(9.41)$ \\
Emotional Stress & $68(7.35)$ \\
Skin and soft tissue infection & $61(6.59)$ \\
Any recent surgical intervention $(<1$ month) & $21(2.27)$ \\
Acute renal failure & $21(2.27)$ \\
Acute coronary syndrome (AMI) & $19(2.05)$ \\
Trauma & $7(0.76)$ \\
Others & $925(100.00)$ \\
Total &
\end{tabular}

Table 3 below shows the frequency and reasons for recurrent ketoacidosis and discontinuation of insulin. The majority of the patients $(65.30 \%)$ had two or more episodes of DKA and the main reasons for recurrent diabetic ketoacidosis were infectious illness $(69.50 \%)$ and discontinued insulin $(14.80 \%)$. Whereas the main reason for discontinued insulin was fade-up of daily injection (47.17\%).

Table 3. Frequency and reasons for recurrence of DKA and discontinuation of insulin among adult DKA patients admitted to AHMC emergency department and medical wards, since they diagnosed as diabetes, Oromia region, Ethiopia, 2017.

\begin{tabular}{ll}
\hline Variables & Frequency (\%) \\
\hline Frequency of recurrent ketoacidosis & $110(30.80)$ \\
One & $172(48.20)$ \\
Two & $61(17.10)$ \\
$\geq$ Three & $14(3.90)$ \\
No previous episodes of DKA & $357(100.00)$ \\
Total & \\
Reasons for recurrent diabetic ketoacidosis & $53(14.80)$ \\
Discontinued insulin & $248(69.5)$ \\
Infectious illness & $42(11.80)$ \\
Other medical conditions & $14(4.00)$ \\
Others & $357(100.00)$ \\
Total & \\
Reasons for discontinued insulin & $20(37.73)$ \\
Lack of supplies & $25(47.17)$ \\
Fade up of daily injection & $8(32.00)$ \\
Combination of reasons & $53(100.00)$ \\
Total &
\end{tabular}

Polyuria/Polydepsia (19.03\%) was reported by most DKA patients followed by the nausea/vomiting (16.17\%). Nearly three -quarter of patients $((68.67 \%)$ had signs of dehydration whereas constitutional signs in nearly one sixth $(18.68 \%)$ of the DKA patients. On average, patients temperature was 
$\left(36.87 \pm 0.77^{\circ} \mathrm{C}\right)$ with mean systolic and diastolic blood pressure was $118.54 \pm 19.53 \mathrm{mmHg}$ and 76.46 (12.02) $\mathrm{mmHg}$ respectively. In addition the average pulse rate of DKA patients was $92.22 \pm 18.92$ beats/min (Table 4 ).

Table 4. Physical presentation of DKA patients admitted to AHMC emergency department and medical wards, Oromia region, Ethiopia, 2017.

\begin{tabular}{ll}
\hline Variable & Frequency (\%) \\
\hline Symptoms of DKA & \\
Polyuria/Polydepsia & $319(19.03)$ \\
Nausea/Vomiting & $271(16.17)$ \\
Weight loss & $236(14.08)$ \\
Malaise & $220(13.13)$ \\
Abdominal Pain & $203(12.11)$ \\
Anorexia & $179(10.68)$ \\
Shortness of Breath & $141(8.41)$ \\
Legs cramp & $54(3.22)$ \\
Loss of conscious & $27(1.61)$ \\
Constitutional Symptoms & $26(1.55)$ \\
Total & $1676(100)$ \\
Sign of DKA & \\
Dehydration & $445(68.67)$ \\
Constitutional signs & $102(15.74)$ \\
Cardiac arrhythmia & $54(8.33)$ \\
Ketosis breath & $47(7.25)$ \\
Total & $648(100)$ \\
& Mean $\pm(\mathrm{SD})$ \\
Temperature $\left({ }^{\circ} \mathrm{C}\right)$ & $36.87( \pm 0.77)$ \\
Blood pressure systolic $(\mathrm{mmHg})$ & $118.54( \pm 19.53)$ \\
Blood pressure diastolic $(\mathrm{mmHg})$ & $76.46( \pm 12.02)$ \\
Pulse rate (beats/min) & $92.22( \pm 18.92)$ \\
\hline
\end{tabular}

The average random blood glucose level, serum potassium, WBC count and hemoglobin was 428.94 120 $\mathrm{mmol} / \mathrm{L}, 3.74 \pm 1.22 \mathrm{mmol} / \mathrm{L}, 11.39 \pm 8.49\left(10^{3} / \mu \mathrm{L}\right)$ and $13.68 \pm 2.39 \mathrm{~g} / \mathrm{dL}$ respectively (Table 5 ).

Table 5. Biochemical, Renal function, Electrolyte and WBC profiles of DKA patients admitted to AHMC emergency department and medical wards, Oromia region, Ethiopia, 2017.

\begin{tabular}{ll}
\hline Variable & Value (mean \pm SD) \\
\hline Random Blood Glucose Level mmol/L & $428.94 \pm 120$ \\
Renal function test & \\
Serun creatinin & $0.89 \pm 0.44$ \\
serum BUN & $30.31 \pm 19.94$ \\
Serum Sodium $\mathrm{mmol} / \mathrm{L}$ & $133.02 \pm 7.35$ \\
Serum Potassium $\mathrm{mmol} / \mathrm{L}$ & $3.74 \pm 1.22$ \\
WBC Count $\mathrm{x} 10^{3} / \mu \mathrm{L}$ & $11.39 \pm 8.49$ \\
RBC Count $\mathrm{x} 10^{6} / \mu \mathrm{L}$ & $4.53 \pm 0.84$ \\
Hemoglobin $\mathrm{g} / \mathrm{dL}$ & $13.68 \pm 2.39$ \\
Hematocrit $\%$ & $40.28 \pm 6.90$ \\
Platelets $\mathrm{x} 10^{3} / \mu \mathrm{L}$ & $268.62 \pm 107.43$ \\
\hline
\end{tabular}

Concerning urine ketones level, nearly half of the patients $(49.30 \%)$ had more than or equal +3 urine ketone level (figure 1).

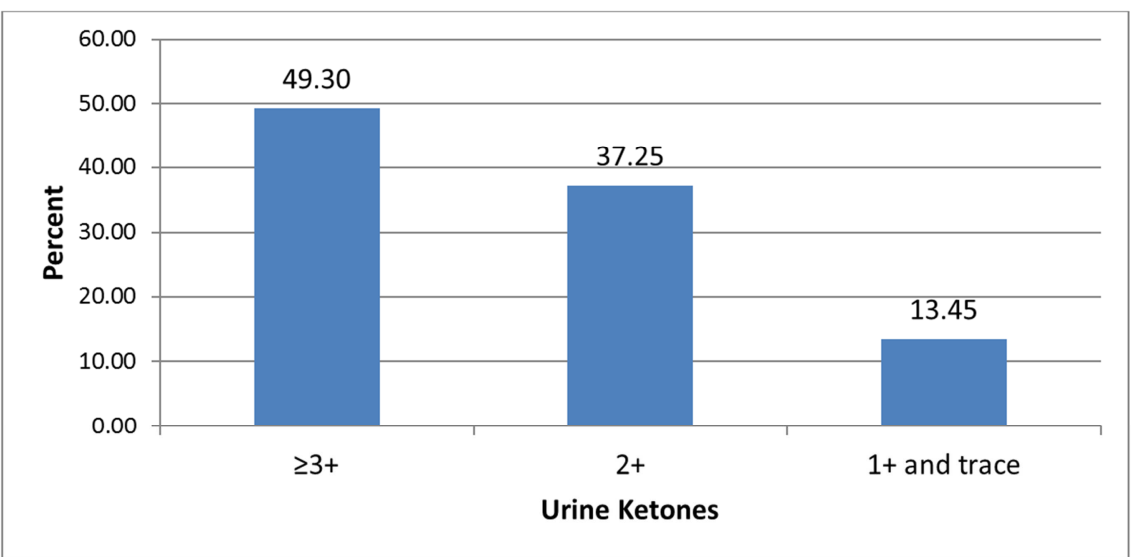

Figure 1. Distribution of urine ketones grades of DKA patients admitted to AHMC emergency department and medical wards, Oromia region, Ethiopia, 2017.

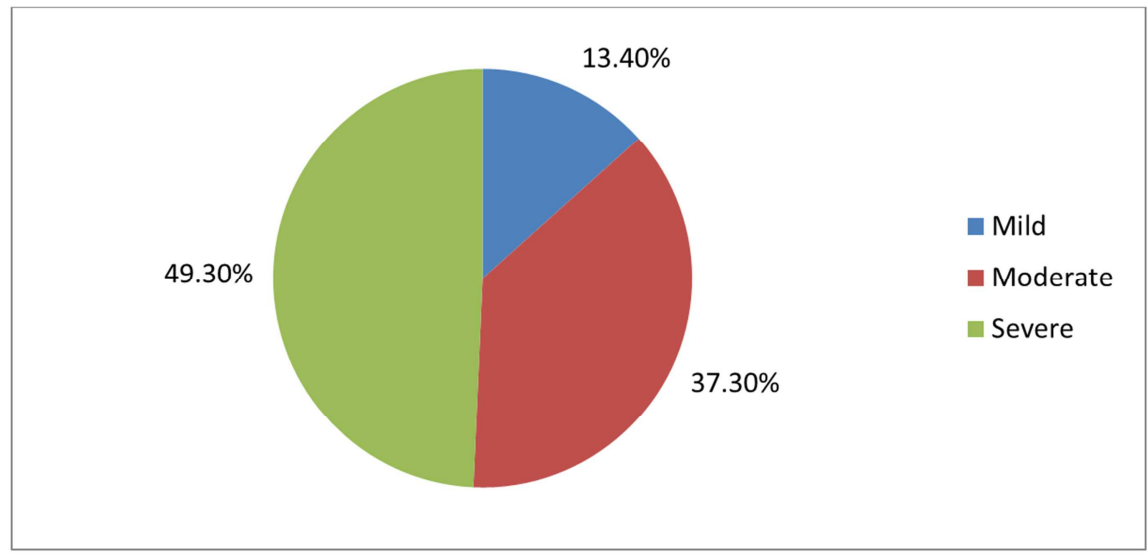

Figure 2. Severity of DKA Episode patients admitted to AHMC emergency department and medical wards, Oromia region, Ethiopia, 2017. 
Concerning management protocol of DKA patients, the most commonly type of fluid used was $0.9 \%$ normal saline $(96.10 \%)$ and majority of total fluid replacement in the 1 st 24 hours was between $3-6$ liters $(49.60 \%)$. The total dose of insulin administration in the 1 st 24 hours, majority of them administered (40.90\%) 40-60 international unit. Similarly, majority of patients $(35.00 \%)$ get out of DKA within less than 5 hours of treatment initiation. More than half of the patients were started with intermediate acting insulin overlapped on the regular insulin soon after the patients get stabilized and start to feed. Due to failure to determine serum potassium level only $39.80 \%$ of patients got potassium supplement. With regard to treatment outcomes of DKA patients, majority of them $(84.90 \%)$ showed improvement and discharged (Table 6).

Table 6. Management protocols of DKA patients admitted to AHMC emergency department and medical wards, Oromia region, Ethiopia, 2017.

\begin{tabular}{ll}
\hline DKA management protocols & Frequency (\%) \\
\hline Types of fluid used in the 1st 24 hours & \\
$0.9 \% \mathrm{NS}$ & $343(96.10)$ \\
$5 \% \mathrm{DW}$ & $14(3.90)$ \\
Total & $356(100.00)$ \\
Total fluid replacement in the 1st 24 hours & \\
$\leq 3$ liters & $97(27.20)$ \\
$3-6$ liters & $177(49.60)$ \\
$>6$ liters & $83(23.20)$ \\
Total & $357(100.00)$ \\
Insulin administration (total RI required in the $1^{\text {st }} 24$ & \\
hours) & \\
$<40$ IU & $83(23.20)$ \\
$40-60$ IU & $146(40.90)$ \\
$>60$ IU & $128(23.20)$ \\
Total & $357(100.00)$ \\
Time within which patient get out of DAK & $125(35.00)$ \\
$<5$ hours & \\
\hline
\end{tabular}

\begin{tabular}{ll}
\hline DKA management protocols & Frequency (\%) \\
\hline $5-12$ hours & $119(33.30)$ \\
$>12$ hours & $113(31.70)$ \\
Total & $357(100.00)$ \\
$\begin{array}{l}\text { Intermediate acting insulin overlapped soon after } \\
\text { patient get stabilized }\end{array}$ & \\
Yes & $142(39.80)$ \\
No & $215(60.20)$ \\
Total & $357(100.000$ \\
Potassium replacement & \\
Yes & $155(43.40)$ \\
No & $202(56.600$ \\
Total & $357(100.00)$ \\
Reasons for not Potassium replacement done & \\
No serum potassium done & $188(93.06)$ \\
Other reasons & $14(6.94)$ \\
Total & $202(100.00)$ \\
General treatment outcomes of DKA & \\
Shows improvement and discharged & $303(84.90)$ \\
Not shows improvement (referred or died) & $54(15.10)$ \\
Total & $357(100.00)$ \\
\hline
\end{tabular}

Those DKA patients treated with more than six liters fluid replacement in the 1st 24 hours had better treatment outcome by two times as compared to less than three liters fluid replacement $\quad(\mathrm{AOR}=2.41 \quad(1.58-10.02)$. Similarly, those patients who got more than sixty international unit insulin doses in the 1 st $24 \mathrm{hrs}$ had better treatment outcome by ten times $(\mathrm{AOR}=10.68$ (3.88- 20.64)) when compared to less than forty international unit insulin administration. In addition, DKA patients who got supplemental potassium showed five times treatment outcome improvement $(\mathrm{AOR}=$ $5.30(2.11-13.32)$ than for those potassium replacement was not done (Table 7).

Table 7. Factors associated with a treatment outcome of DKA patients admitted to AHMC emergency department and medical wards, Oromia region, Ethiopia, 2017.

\begin{tabular}{|c|c|c|}
\hline \multirow[t]{2}{*}{ DKA Treatment } & \multicolumn{2}{|c|}{ Management outcomes of DKA } \\
\hline & COR (95\% CI) & AOR (95\% CI) \\
\hline \multicolumn{3}{|c|}{ Fluid replacement in the 1 st 24 hours } \\
\hline$<3$ liters & 1 & 1 \\
\hline 3-6 liters & $3.60(1.34-9.64)$ & $2.42(0.75-7.81)$ \\
\hline$>6$ liters & $5.84(2.08-16.37)$ & $2.41(1.58-10.02)$ \\
\hline \multicolumn{3}{|c|}{ Insulin administration in the 1 st 24 hours } \\
\hline$<40$ IU & 1 & 1 \\
\hline$>60 \mathrm{IU}$ & $7.14(2.43-21.00)$ & $10.68(3.88-20.64)$ \\
\hline \multicolumn{3}{|c|}{$\begin{array}{l}\text { Intermediate acting insulin overlapped soon after patient get } \\
\text { stabilized }\end{array}$} \\
\hline No & 1 & 1 \\
\hline Yes & $3.91(1.84-8.28)$ & $12.96(4.88-34.37)$ \\
\hline \multicolumn{3}{|c|}{ Potassium replacement } \\
\hline No & 1 & 1 \\
\hline
\end{tabular}

\section{Discussions}

Lifelong co-morbidities of metabolic continuum are mostly associated with DM. More than half of the DKA patients had one or more of the chronic complications, which is similar with the results from Jimma University specialized hospital [24]. The major one was Hypertension (29.85\%), which is slightly higher than the reported from Jimma University specialized hospital (24.9\%), followed by diabetic 
foot ulcer (18.21\%) which was lower than a study done in Tikur Anbessa Hospital 35\% [23]. Similarly, in our study sexual dysfunctions was $13.73 \%$, this was much lower than the report from Tikur Anbessa and St. Paul's University Teaching Hospital (55\%) [22], the discrepancy may be due to under reporting of cases in this study.

Concerning acute diabetic complication, all of the patients were documented at least one acute co-morbidities, where urinary tract infection was the commonest $(22.59 \%)$. This finding was higher than a study done in Tikur Anbessa Hospital reported urinary tract infection (UTI) was 14\% [23]. This discrepancy also may be due to missing reports.

In our research, majority of patients had two or more episodes of DKA indicating poor glycemic control and high risk of mortality and morbidity from acute complications and the main reasons for recurrent diabetic ketoacidosis were infectious illness and drug discontinuation. Patient education regarding infection prevention, advice to visit their physician soon they get sick and adjusting their insulin dose as per their blood sugar during stressful condition needs to be emphasized. Similarly the main reason for discontinuing insulin was fade-up of daily injection $(47.17 \%)$. This is in line with among the precipitating factors for an episode of DKA, infection and nonadherence to insulin are, more or less, equally reported in other findings $[6,20]$.

Nearly three -quarter of patients had signs of dehydration and acidosis implying severe form of DKA and high mortality, which was consistent with the study done in Jimma University Specialized Hospital diabetes follow-up clinic (64\%) [21].

The average random blood glucose level was $428.94 \pm 120$ $\mathrm{mmol} / \mathrm{L}$, which is far higher than the recommendations values $(<250(\mathrm{mg} / \mathrm{dL})$ and considered as a severe episode of DKA [1]. The reasons may be poor nutritional status, physical exercise and drug adherence. Almost half of the DKA patients $(49.30 \%)$ had more than or equal +3 urine ketone level, it is an indicator of severe DKA level with high mortality [23], which needs argent managements.

Concerning management protocol of DKA patients in our study, the most commonly type of fluid used was $0.9 \% \mathrm{NS}$ $(96.10 \%)$ and majority of total fluid replacement in the $1^{\text {st }} 24$ hours was between $3-6$ liters $(49.60 \%)$. This is lower than the recommended standard value $\left(5-7\right.$ liters in the $1^{\text {st }} 24$ hours). Correct with normal saline, 2liters in the first 2 hours, the remainder over the next 22 hours [1]. Further study is needed for the less volume of fluid replacement.

Similarly, dose of regular insulin administration in the $1^{\text {st }}$ 24 hours was slightly lower than different recommendations $[1,23]$. The recommended value explain that, intravenous infusion bolus of $0.1 \mathrm{IU} / \mathrm{Kg}$ regular insulin followed by an intravenous infusion of $0.1 \mathrm{IU} / \mathrm{kg} / \mathrm{h}$. Goal is to reduce plasma glucose by $50-75 \mathrm{mg} / \mathrm{dL} / \mathrm{h}$. Initial target plasma glucose is 200-250 $\mathrm{mg} / \mathrm{dL}$. Once achieved, reduce insulin rate and provide dextrose to 'clamp' the plasma glucose until acidosis/anion gap resolved.

Regarding electrolytes replacement, potassium replacement was done only for less than half the patients
$(39.80 \%)$ and the main reasons for suboptimal potassium replacement was absence of serum potassium determination. But different finding suggested that close monitoring and correction of serum potassium is critical. If serum potassium is less than 3.5, correct hypokalemia before any insulin is given $[4,5]$.

Finally about treatment outcomes of DKA patients, majority of them $(84.90 \%)$ showed improvement and discharged. This is higher than the study finding from Jimma University Specialized hospital; where $71.10 \%$ was discharged with improvements [24]. The difference may be due to different quality of services given at each institution.

Those DKA patients treated with more than six liters fluid replacement in the 1st 24 hours had improved treatment outcome by two times than less fluid replacement $(\mathrm{AOR}=2.41$ (1.58-10.02). This finding is comparable with WHO recommendation, which stated that, initially fluid therapy is aimed at rapid restoration of the intravascular volume. In the first hour $0.9 \%$ saline is given at a rate of 15 $20 \mathrm{mls} / \mathrm{kg}$ or an average 1-1.5 liters. Thereafter further fluid therapy should be administered at a rate sufficient to maintain adequate blood pressure, urine output and mental status. The aim is to correct the estimated water deficit over 24hours; in general a rate of $4-14 \mathrm{mls} / \mathrm{kg} /$ hour will suffice patients in cardiogenic shock will require inotropes and haemodynamic monitorin $[1,33]$.

Similarly, more than sixty IU insulin administration in the 1st 24 hours, improved treatment outcome by ten times $(\mathrm{AOR}=10.68$ (3.88- 20.64)) than less insulin administration. This is also in line with different recommendations which states; once hypokalaemia has been excluded an intravenous insulin infusion should be commenced at 0.1 units $/ \mathrm{kg} /$ hour [3, 4].

In addition, DKA patients whom potassium replacement was done showed five times treatment outcome improvement $(\mathrm{AOR}=5.30$ (2.11-13.32) (Table 4). Different findings also support that, adding $20-40 \mathrm{mmol} / 1 \mathrm{KCl}$ will usually result in adequate replacement, keeping the serum potassium around $4.5 \mathrm{mmol} / 1[6,8]$.

\section{Limitation of the Study}

1. The major limitation was poor chart keeping that might have limited us from getting full information about associated factors of DKA treatment outcome.

2. The study findings was only limited to one hospital (Adama Hospital Medical College) and other hospitals in the region were not incorporated in this study. Therefore, research results are limited to this particular institution and cannot be generalized to other institutions in Oromia.

\section{Conclusion and Recommendations}

In conclusion,

1. Majority of patients had two or more episodes of DKA indicating poor glycemic control and high risk of 
mortality and morbidity from acute complications.

2. The main reasons for recurrent diabetic ketoacidosis were infectious illness and insulin discontinuation.

3. DKA Treatment outcome is significantly associated with amount of fluid replacement, insulin administration and potassium replacement

4. Finally, majority of DKA patients treated at Adama Hospital Medical College emergency room and medical wards were discharged with improvement.

Recommendations

1. Early treatment of infection, fluid replacement and insulin dose adjustment during illness need to be encouraged as per the recommendation of local and international guidelines for the care of diabetic patients and management of acute metabolic complications.

2. Especially, more emphasis should be given to raise the awareness of the general public about insulin adherence and over all comprehensive diabetic care

3. A further clinical investigation for adequacy of treatment outcome and associated factors of DKA with appropriate prospective study design is also warmly recommended.

\section{References}

[1] Kitabchi AE, Umpierrez GE, Murphy MB, et al. Management of hyperglycemic crises in patients with diabetes. Diabetes Care. 2001; 24 (1):131-53.

[2] Wolfsdorf J, Glaser N, Sperling MA. Diabetic ketoacidosis in infants, children, and adolescents: a consensus statement from the American Diabetes Association. Diabetes Care 2006; 29:1150-2259.

[3] Kitabaci AE, Umpierrez GE, Murphy MB, Kreisberg RA. Hyperglycaemic crises in adult patients with diabetes. A consensus statement from the American diabetes association. Diabetes Care 2006; 29: 2739-2748.

[4] Mbugua PK, Otieno CF, Kayima JK, Amayo AA, McLigeyo SO. Diabetic ketoacidosis: Clinical presentation and precipitating factors at Kenyatta National Hospital, Nairobi. East African Medical Journal 2005; 82 (12 Suppl):S191-6.

[5] Alvin C. powers. Diabetes mellitus. In: Harrison, Brauwnwald, Kasper (eds.) Harrison's Principles of Internal Medicine, 17th edition, The McGraw-Hill Companies, Inc 2008: 2275-304.

[6] Otieno CF, Kayima JK, Omonge EO, Oyoo GO. Diabetic ketoacidosis: risk factors, mechanisms and management strategies in sub-Saharan Africa: a review. East Afr Med J 2005; 82: S197-203.

[7] http://www.bsped.org.uk/professional/guidelines/docs/ DKAGuideline.pdf Last accessed 20 January 2017.

[8] The-Management-of-Diabetic-Ketoacidosis-in-Adults/; http:// www.diabetes.nhs.uk/publications and resources/reports. Accessed 30 June 2017.

[9] Oyarzabal Irigoyen M, etals. Ketoacidosis at onset of type 1 Diabetes mellitus in pediatric age in Spain and review of the literature. Pediatr Endocrinol Rev. 2012 Mar; 9 (3):669-71.).
[10] http://www.cdc.gov/nchs/ about/major/hdasd/nhds.htm. Accessed 24 June 2017.

[11] http://www.tjpr.org http://dx.doi.org/10.4314/tjpr.v14i1.25. Accessed 25 June 2017.

[12] Chaithongdi N, Subauste JS, Koch CA, Geraci SA. Diagnosis and management of hyperglycemic emergencies. Hormones (Athens). 2011 Oct-Dec; 10 (4):250-60.

[13] Goldberger ZD. Severe hypothermia with Osborn waves in diabetic ketoacidosis. Respir Care 2008; 53:500-2.

[14] Umpierrez G, Freire AX. Abdominal pain in patients with hyperglycemic crises. J Crit Care 2002; 17:63-67.

[15] Chaithongdi N, Subauste JS, Koch CA, Geraci SA. Diagnosis and management of hyperglycemic emergencies. Hormones (Athens). 2011 Oct-Dec; 10 (4):250-60.

[16] Kitabchi AE, Umpierrez GE, Fisher JN, Murphy MB, Stentz FB. Thirty years of personal experience in hyperglycemic crises: diabetic ketoacidosis and hyperglycemic hyperosmolar state. J Clin Endocrinol Metab. 2008 May; 93 (5):1541-52.

[17] Kitabchi AE, Murphy MB, Spencer J, Matteri R, Karas J. Is a priming dose of insulin necessary in a low-dose insulin protocol for the treatment of diabetic ketoacidosis? Diabetes Care. 2008 Nov; 31 (11):2081-5.

[18] Umpierrez GE, Latif K, Stoever J, et al, Efficacy of subcutaneous insulin lispro versus continuous intravenous regular insulin for the treatment of patients with diabetic ketoacidosis. Am J Med 2004; 117: 291-296.

[19] Della Manna T, Steinmetz L, Campos PR, et al, Subcutaneous use of a fast-acting insulin analog: An alternative treatment for pediatric patients with diabetic ketoacidosis. Diabetes Care 2005; 28: 1856-1861.

[20] Feleke Y, Enquselassie F. An assessment of the health care system for diabetes in Addis Ababa. Ethiop. J Health dev. 2005; 19 (3): 203-210.

[21] Gudina et al, Assessment of quality of care given to diabetic patients at Jimma University Specialized Hospital diabetes follow-up clinic, Jimma, Ethiopia, BMC Endocrine Disorders 2011.

[22] Adem A, Demis T, Feleke Y. Trend of diabetic admissions in Tikur Anbessa and St. Paul's University Teaching Hospitals from January 2005-December 2009, Addis Ababa, Ethiopia. Ethiop Med J. 2011; 49 (3):231-8.

[23] Seblework Temsegen, Acute Management of Diabetic Ketoacidosis in Tikur Ambesa Hospital's Adult Emergency department and medical wards, American International Health Alliance's, 2012.

[24] Dawit Worku (MD), Leja Hamza (MD), Kifle Woldemichael (MD), patterns of diabetic complications at Jimma university specialized hospital, southwest Ethiopia, Ethiop J Health Sci. Vol. 20, No. 1 March 2010.

[25] Kitabchi AE, Umpierrez GE, Miles JM, Fisher JN. Hyperglycemic crises in adult patients with diabetes. Diabetes Care 2009; Jul; 32 (7):1335-43.

[26] Weissberg-Benchell J, Antisdel-Lomaglio J, Seshadri R. Insulin pump therapy: a meta-analysis. Diabetes Care 2003; 26: 1079-1087. 
[27] Peterson LN, Levi M. Disorder of potassium and metabolism. In: Schrier RW (ed). Renal and electrolyte disordes, 6th edn, Lippincott Williams \& Wilkins, Philadelphia; 2002; pp, 171215.

[28] Calzavacca P, Lacari E, Bellomo R. In: Stewart's Textbook of Acid-Base. Ebers P, Kellum J Lulu.com, editor. 2009. Renal failure; pp. 394-395. Clinical applications of quantitative acid-base medicine.

[29] Kraut JA, Kurtz I. Use of base in the treatment of severe acidemic states. American Journal of Kidney Diseases 2001; 38:703-727.

[30] Eisenbarth GS, Polonsky KS, Buse JB 2008 Acute diabetic emergencies: Diabetic ketoacidosis. In: Kronenberg HM, Melmed S, Polonsky KS et al (eds). Williams Textbook of
Endocrinology, 11th edn, Saunders Elsevier, Pennsylvania; pp, 1407-1416.

[31] Geerse DA, Bindels AJ, Kuiper MA, et al, Treatment of hypophosphatemia in the intensive care unit: a review. Crit Care 2010; 14: R147.

[32] Umpierrez GE, Jones S, Smiley D, Mulligan P, Keyler T, Temponi A, Semakula C, Umpierrez D, Peng L, Cero'n M, Robalino G. Insulin analogs versus human insulin in the treatment of patients with diabetic ketoacidosis: a randomized controlled trial. Diabetes Care. 2009 Jul; 32 (7):1164-9.

[33] Arefi M, Tabrizchi N. Nalidixic acid and diabetic ketoacidosis. Indian J Endocrinol Metab. 2012 Mar; 16 Suppl 1:S124-6. 